\title{
Medicina Interna, paziente complesso... verso una clinica relazionale e ragionevole
}

\section{Clinic, complex and complicated patient, relations and connexions}

\author{
Ivan Cavicchi*
}

Docente di Sociologia e di Filosofia della Medicina, Facoltà di Medicina e Chirurgia, Università Tor Vergata, Roma

Ricevuto il 7 settembre 2012; accettato l'1 ottobre 2012

Disponibile online il 23 ottobre 2012

\section{KEYWORDS}

Complex patient;

Evidence based

medicine;

Internal medicine.
Summary To know the complexity of patient the clinic should think about the relations of complexity which can bond scientific evidence and doctor and patient. To think and to act according to the relations means to settle a clinic, pragmatic and reasonable rational. (c) 2012 Elsevier Srl. All rights reserved.

\section{Introduzione}

Lo spunto per questo contributo è venuto da un articolo [1] di grande interesse per le seguenti ragioni:

- si avanza una critica a un certo proceduralismo, cioè a un modo di conoscere clinico prevalentemente "basato" su astrazioni statistiche e linee guida;

- tale critica riguarda prevalentemente la Evidence Based Medicine (EBM) ma vale anche per una forma particolare di verificazionismo, cioè per quei criteri che in clinica sinora hanno stabilito la demarcazione tra ciò che è giusto e ciò che non lo è, tra quello che si deve fare e quello che non si deve fare. L'EBM è un criterio di demarcazione su base statistica con l'ambizione di sostituire quelli osservazionali/empirici/fattuali della clinica classica;

- si riammettono metaforicamente le non evidenze, cioè i criteri osservazionali/empirico/fattuali della clinica, e si ammette, quale forma complementare di conoscenza, l'opinione del malato. La tradizionale distinzione tra episteme e doxa viene meno, fino a configurare una sorta di teoria delle preferenze del malato;

- le ricadute di questi importanti ripensamenti, seppur in nuce, aprono la possibilità di una ridiscussione su cosa deve essere la clinica oggi. Essi prendono forma a partire da una nuova idea di complessità, che viene considerata non più un limite alla conoscenza clinica, cioè un ostacolo da superare con alcune semplificazioni, ma un suo fondamentale presupposto;

- si sostiene che il malato complesso è irriducibile ai criteri proceduralisti dell'EBM e delle linee guida.

L'articolo fondamentalmente rappresenta una critica importante all'EBM, che, malgrado le sue iniziali promesse riformatrici, non si è rivelata il ripensamento che si sperava. L'EBM tuttavia resta una fonte importante di conoscenza, come del resto qualsiasi informazione epidemiologica, ma a condizione di liberarsi di quelle velleità e rigidità che gli

\footnotetext{
* Corrispondenza: via dei Vascellari 28 - 00153 Roma, Italia.

E-mail: info@ivancavicchi.it (I. Cavicchi).
} 
epigoni di Sacket hanno incautamente cavalcato e che molti anni fa confutai non senza dissensi e incomprensioni. L'articolo ha importanti implicazioni sull'ortodossia dottrinale, collocando la clinica in una posizione davvero interessante "tra ciò che non è più" e "ciò che non è ancora", o meglio "tra ciò che è ancora" e "ciò che dovrebbe diventare". L'intento di questo articolo è contribuire a definire meglio l'evoluzione dell'idea di clinica. Indubbiamente l'EBM tradisce vistose aporie nei confronti del malato complesso, tuttavia questo non giustifica un semplice ritorno all'ortodossia clinica per rimuoverle. L'EBM non è nata per caso, essa ha tentato, in un momento particolare della medicina, con una teoria della rappresentazione statistica del malato, di rispondere a nuove complessità sociali, culturali, economiche. Il tentativo si è come infranto contro le realtà complesse che la clinica deve affrontare, e la sua credibilità si è parecchio ristretta, ma le complessità, nei confronti della clinica, rimangono tutte.

\section{Definizione di complessità}

La parola "complessità" è giovane, quasi un neologismo. Essa nasce aggiungendo all'aggettivo "complesso" il suffisso "ità". In questo modo ciò che è complesso viene de-aggettivato e trasformato in sostantivo. "Complessità" compare nei vocabolari a partire dagli anni Cinquanta/Sessanta, dopo che la biologia aveva iniziato il suo profondo ripensamento epistemologico, ed esploderà, quasi come una moda, negli anni Settanta/Ottanta. La famosa "sfida della complessità". Perché complessità? La biologia scopre che il vivente ha una sua organizzazione, quindi una natura sistemica. L'aggettivo "complesso", che ha sempre definito la qualità del biologico, per la prima volta diventa un oggetto di studio e di conoscenza, cioè una realtà da conoscere. Quindi cos'è la complessità? È una nuova realtà ontologica, cioè un'entità con propri predicati, aggettivi, attributi e qualità [2]. Da questo momento in poi la complessità diventa, o sarebbe dovuta diventare, la condizione per conoscere la realtà. Se la realtà è coemergenza di una complessità, cioè di un'organizzazione sistemica sottostante, essa andrebbe ridefinita e ripensata attraverso e per mezzo di tale organizzazione sistemica, perché è la realtà che la esprime. In ragione di ciò per la medicina, ma non solo, sorge la necessità di un ripensamento profondo.

\section{Malato complesso e malato complicato}

Il malato è "complesso" in quanto realtà molteplice e multidimensionale. Egli, in quanto tale, è un'unità molteplice costituita da tante cose: un essere, una persona, un fenomeno, un contesto [3]. Conoscere il malato attraverso la sua complessità significa:

- avere una sensibilità ontologica nei confronti dell'essere;

- confrontarsi con le opinioni della persona;

- conoscere biologicamente la malattia come fenomeno;

- tenere conto di un contesto, cioè dei servizi in cui si opera, dei problemi di spesa, dei diritti, dei rapporti con altre professioni ecc.

Questa è la complessità. Altra cosa è la definizione di "malato complesso" fornita dall'Agency for Healthcare Research and Quality (AHRQ), cioè: "Una persona affetta da due o più malattie croniche, in cui ciascuna delle condizioni morbose presenti è in grado d'influenzare l'esito delle cure delle altre coesistenti, attraverso varie modalità: la limitazione della speranza di vita, l'aumentata morbilità intercorrente, le interazioni tra terapie farmacologiche, l'impossibilità del pieno impiego di cure adeguate per controindicazione ecc." [1]. Questa definizione, a ben guardare, definisce la complicazione in quanto la sua logica si limita solo al fenomeno, quindi alla malattia e ragiona come se a "più malattia" corrispondesse "un grado maggiore di complessità". In realtà a "più malattia" corrisponde "un grado maggiore di complicazione". In questa definizione, cioè, sono inclusi, per quanto accentuati, i condizionali della malattia, mentre restano esclusi quelli che riguardano l'essere, la persona, il contesto. Indubbiamente in un malato anziano, cronico, portatore di più malattie vi è, clinicamente parlando, un grado di complicazione in più. È l'accresciuta complicazione patologica che vanifica la pretesa scientificità dell'EBM e delle linee guida e che obbliga il clinico a tornare a un'epistemologia osservazionale, empirica, congetturale. Ma se la complicazione mette fuori gioco l'EBM, figuriamoci la complessità. Il che vuol dire che non solo per il "malato complesso", ma per ogni malato, l'EBM va usata con molta cautela, vale a dire con la minore dogmaticità possibile. L'ingenuità e la pretesa dell'EBM sono state, da una parte, ridurre la complessità a una teoria della rappresentazione statistica del malato e, dall'altra, negare il problema della complicazione cioè il "mondo reale" dei malati [4]. In effetti il malato complesso è qualcosa che coemerge da una ordinaria complessità ontologica del malato, più una maggiore complicazione clinica. Il grado di complicazione clinica non è però sufficiente a definire la complessità di un malato. Essa resta una parte dell'intero, ma non è l'intero.

\section{Teoria della congiunzione}

La clinica sa come conoscere il malato complicato, sa che deve in qualche modo de-proceduralizzare il suo giudizio, e sa che il modo migliore è navigare a vista e decidere il cammino passo dopo passo mettendosi in gioco come clinica. Ma come fa un clinico a conoscere la complessità? Se ragionassimo con la logica giusta si tratterebbe di interconnettere tra loro tutte le variabili principali che la compongono. Cioè si tratta di ri-comporre il malato come "sistema di parti", non di de-comporlo come "insieme di parti". Il sistema non è mai solo un insieme, cioè solo la somma delle parti. Per interconnettere le tante parti di un malato la categoria chiave è relazione. La relazione, da un punto di vista epistemologico, è una specie di ponte che collega mondi visti tradizionalmente in modo separato, quindi è una "e", cioè una congiunzione [5]. I termini diversi di un malato uniti e riuniti dalla "e" ricostruiscono il suo grado di complessità. La congiunzione che oggi la clinica deve saper coniugare riguarda fondamentalmente due nozioni: il mondo dell'evidenza scientifica, quindi le verità biomediche della malattia, "e" il mondo dell'essere e della persona dentro un contesto non casuale. La "conoscenza clinica" della malattia diventa, con una "e", conoscenza scientifica. . . e altra conoscenza, quindi conoscenza dell'organo e conoscenza del soggetto, conoscenza biologica e conoscenza relazionale ecc. La conoscenza diventa un reticolo di congiunzioni, cioè di conoscenze di natura diversa. 


\section{Evidenza scientifica $e$ conoscenza clinica}

C'è un grande equivoco da chiarire: la medicina da sempre cerca e si avvale di quelle che si definiscono evidenze scientifiche; la pretesa dell'EBM è di aver proposto di sostituire i tanti tipi di evidenze scientifiche con una evidenza statistica, che in qualche modo le riassumesse tutte.

Se di evidenze ve ne sono tante (osservazionali, empiriche, fattuali, contingenti, organiche, biochimiche ecc.) le evidenze scientifiche sono solo quelle accertate attraverso procedure di descrizione, misurazione, valutazione, controllabilità. L'abbaglio che hanno preso i teorici dell'EBM è stato di credere che le evidenze statistiche potessero vicariare le evidenze cliniche. II malato complicato e la complessità del malato hanno dimostrato ormai che questa vicarianza non può essere data. L'EBM induttivamente generalizza certe astrazioni statistiche elaborate con metodologie metanalitiche e ha tutti i pregi e i difetti delle astrazioni statistiche e delle generalizzazioni. Diversamente, l'evidenza clinica si avvale di tutto quanto è in grado di dimostrare una verità plausibile (esperienza, tecnologia, esperimenti, osservazione, constatazioni fattuali, conoscenze biochimiche, relazioni, situazioni, anamnesi, procedure diagnostiche) e si avvale di diverse logiche, inferenze, ragionamenti deduttivi, induttivi, pratico-deduttivi, abduttivi, in grado di dimostrare con ragionevole scientificità qualcosa [6].

L'assimilazione dell'EBM all'evidenza scientifica è stata favorita da una confusione terminologica: la nozione di "evidenza" (clearness, truth, obviousness) è stata confusa con la nozione di "prova" (evidence, proof). La prova sta all'evidenza come un mezzo sta a uno scopo, cioè come un dato sta alla conoscenza.

In ragione della complessità del malato la clinica ha imparato a essere prudente. Può darsi che di fronte a certe prove, soprattutto diagnostiche e di laboratorio, essa finisca per assumerle direttamente come evidenze. In questi casi vale la regola transitiva: se in una radiografia compaiono delle metastasi, allora le metastasi sono la prova di una probabile evidenza oncologica. Ma di fronte a eventi non chiari e ambivalenti, di fronte al malato complicato, la regola transitiva tra prova ed evidenza non si può usare. In particolare, non è possibile usare la regola transitiva tra prove statistiche ed evidenze cliniche. Per un clinico le prove, comprese quelle utilissime della statistica epidemiologica, rappresentano semplicemente dati interpretabili che, a loro volta, lo obbligano a un supplemento d'indagine per cercare più soddisfacenti evidenze scientifiche.

Tuttavia, EBM a parte, oggi la questione dell'evidenza scientifica in quanto tale merita di essere ripuntualizzata proprio rispetto ai condizionali di complessità. La natura razionale ed empirica della clinica ha sempre visto l'evidenza scientifica come "una verità irresistibile", cioè come indiscutibile constatazione dei fatti, ma oggi i fatti spesso tradiscono un'oggettività relativa a tanti fattori, a partire dalle caratteristiche dell'osservatore clinico. Inoltre, l'innegabile base ipotetica dei ragionamenti medici oggi non può che guardare all'evidenza all'interno del problema della conferma e della probabilità diagnostica, quindi all'interno della giustificazione epistemica [7]. In fin dei conti in clinica non esiste un'evidenza tout-court. L'evidenza, nella maggior parte dei casi, serve a determinare se e in quale misura un sintomo può valere come evidenza per un'ipotesi di malattia, oppure per mettere a confronto informazioni biomediche diverse e valutare l'ipotesi più probabile; o, ancora, per stabilire il livello in cui una certa informazione biomedica conferma una certa ipotesi. In sostanza, proprio in ragione della complessità, si tende a revocare all'evidenza la possibilità di essere definita con criteri univoci e di configurarsi come una prova inoppugnabile. Va anche detto che se l'evidenza è sicuramente e sempre una proprietà della malattia, nello stesso tempo e in una certa misura è anche una proprietà del clinico che conosce la malattia. Le componenti oggettive dell'evidenza sono innegabili, quanto quelle doppiamente soggettive del medico e del malato. È proprio in ragione della complessità che per la clinica si tratta di trovare sempre un equilibrio:

un'ipotesi di evidenza (ie) può dirsi evidente per un medico $(m)$ a condizione che ie sia razionale e ragionevole per $m$; che non ci sia alcuna ipotesi $i$ tale che per $m$ sia più razionale e ragionevole credere in $i$ di quanto non lo sia credere in ie.

In questo ragionamento si tenta di equilibrare la dimensione fattuale, oggettiva, constativa dell'evidenza con la dimensione soggettiva del clinico, ma di fatto si ragiona al fine di trovare razionalmente l'evidenza più conveniente. Il malato complesso e complicato non tollera evidenze dogmatiche di alcun tipo: l'evidenza più conveniente è semplicemente quella che rappresenta meglio la sua complessità.

Una volta definita un'evidenza conveniente rimane il problema della relazione con l'opinione del malato:

un'ipotesi di evidenza (ie) può dirsi evidente in una relazione ( $r$ ) a condizione che ie sia razionale/ragionevole per il clinico e per il malato nella relazione $r$; che non ci sia alcuna ipotesi $i$ tale che per il clinico e il malato nella relazione $r$ sia più razionale/ragionevole credere in $i$ di quanto non lo sia credere in ie [8].

\section{Evidenza clinica $e$ credenze del clinico}

Se un medico crede che il suo malato ha una certa malattia, lo fa sulla base della conoscenza di cui dispone. Credere e sapere sono fortemente intrecciati. In senso generale, per chiunque credere significa ritenere probabile o possibile qualcosa. Per un medico, credere che un'evidenza $(e)$ sia vera significa ritenere probabile che le cose siano come lui crede:

se la probabilità è massima, allora credere a un'evidenza significa ritenere con certezza che e sia vera; $e$ in questo caso è considerata un'evidenza scientifica; se la probabilità non è massima, significa che $e$ è solo probabile; in questo caso $e$ è considerata un'evidenza scientifica verosimile.

Se ragioniamo con la logica della complessità, dobbiamo dire che il medico, per avere una qualche evidenza scientifica (es), deve soddisfare almeno quattro condizioni:

1. quella soggettiva della credenza (perché es sia conosciuta occorre che es sia da lui creduta tale);

2. quella oggettiva di verità (che es sia almeno plausibile);

3. quella della giustificazione epistemica (che es sia fondata su un metodo o su un procedimento razionale); 
4. infine, quella dell'opinione del malato (perché es del medico sia conosciuta occorre che sia creduta vera o plausibile anche dal malato).

Se ne deduce che qualsiasi evidenza scientifica non è mai solo il risultato di una verità oggettiva, perché il medico in quella verità deve crederci; non è mai solo il risultato della credenza soggettiva, perché una verità oggettiva bisogna pur averla; e non è mai solo un problema di giustificazione metodologica, perché comunque si tratta di giustificare in un contesto dato delle evidenze ritenute tali. Ma soprattutto non è più solo un problema di conoscenza del medico, perché essa deve comunque essere validata da una relazione con il malato. L'evidenza scientifica rientra in questo modo all'interno delle problematiche delle garanzie del sapere, cioè dentro la necessità per il medico e per il malato di avere un qualche fondamento in cui credere. Sarebbe quindi più corretto parlare di evidenza scientifica condivisa, intendendola come qualcosa che tende a ridurre lo spazio esistente tra ciò che crede il medico, il suo sapere effettivo e ciò che crede il malato e il suo sapere effettivo. Essa, naturalmente, non è priva di fallacie. Non è infrequente che un medico, soprattutto di fronte a malati complessi, creda di avere un'evidenza affidabile e tuttavia possa ingannarsi nel giudicare il tipo di evidenza. Esiste, quindi, anche il problema della possibile inaffidabilità della credenza di evidenza. Insomma la nozione di evidenza scientifica rispetto alla complessità non è così semplice e dimostrativa come sembra.

Del resto la vera evidenza non avrebbe bisogno di essere dimostrata, è autodimostrativa. In clinica non mancano le evidenze autodimostrative, ma nella stragrande maggioranza dei casi le evidenze scientifiche devono essere dimostrate come tali. Una volta l'oggettività delle evidenze scientifiche era garantita dalla metodologia. Oggi, però, la metodologia, particolarmente in regime di complessità, non ha solo il compito di guidare l'indagine medica, ma anche quello di mediare i problemi di che cosa conoscere con $\mathrm{i}$ problemi di colui che conosce e soprattutto con quelli della persona da conoscere. La metodologia, nata come una rigorosa rotaia su cui far camminare la conoscenza, tende oggi a essere una procedura di razionalità accertabile, controllabile, ripetibile che media $\mathrm{i}$ rapporti tra un soggetto che conosce e un soggetto da conoscere [9]. I problemi della metodologia e dell'evidenza scientifica in questa società sono probabilmente $i$ più delicati e pesanti della pratica medica. Le decisioni cliniche si devono confrontare con generi di razionalità diversi da quella scientifica e che rientrano nel mondo delle opinioni, delle culture, delle convinzioni personali. La nuova sfida è la congiunzione tra il mondo della conoscenza scientifica e quello della conoscenza personale.

\section{Evidenza clinica $e$ opinioni del malato}

L'evidenza scientifica per un clinico è verità logica, regola per decidere cosa fare, criterio da cui dipende il come fare. Ma tutto questo oggi deve confrontarsi con le opinioni del malato. Tenere conto delle opinioni del malato è più facile a dirsi che a farsi. Ma non perché, come sostiene noiosamente la letteratura, esiste il problema del paternalismo del medico e quindi quello dell'empowerment del paziente, ma semplicemente perché l'evidenza scientifica in quanto verità tende per sua natura ad autoprescriversi, cioè a imporsi al malato. Per molti medici non ha senso discutere con il malato di verità scientifiche, non perché siano irrispettosi, o paternalisti, o peggio "scientisti", ma solo perché convinti che in medicina le verità scientifiche non andrebbero discusse. In generale ciò che è prescrittivo, in questa società, è visto addirittura come una forma di "disumanità", anche se attuata, come nelle cure mediche, a fin di bene.

Ciò di cui il medico deve convincersi è che oggi è cambiato proprio l'atteggiamento della società nei confronti della razionalità scientifica e della scienza in generale, e quindi nei confronti persino delle evidenze scientifiche. Oggi la società è convinta che la medicina può produrre conseguenze indesiderabili. Da questa consapevolezza nasce la necessità per la clinica di misurarsi con l'opinione delle persone. Alla base dei tanti conflitti tra medicina e società vi è un'evidenza scientifica avvertita come "disumana", perché si occupa solo di corpi e non di persone, e a volte perfino irragionevole nei confronti dei tanti problemi di un malato. La critica principale che la postmodernità rivolge all'evidenza scientifica riguarda soprattutto la questione dell'unilateralità della scelta, cioè l'essere obbligati come malati a certe condotte senza alcuna alternativa. Del resto, per la clinica la scelta obbligata deriva sempre dall'evidenza scientifica. Per il malato postmoderno, l'esigente, la scelta è invece una questione di buon senso, di condivisione e di consensualità, di rispetto. Quindi, per il malato esigente non più paziente qualsiasi evidenza scientifica va condivisa [10].

Evidenza scientifica $e$ opinione del malato è un'altra questione importante del nostro tempo. Se per il clinico si tratta di tenere conto delle "preferenze" delle persone, allora egli non può che essere pratico. Si dice "teorico" il clinico che si pone il problema della correttezza razionale, "tecnico" quello che si pone il problema dei mezzi razionali da impiegare, "pratico" quello che si pone il problema dei fini concreti di una persona malata. Sino a questo momento l'evidenza scientifica è stata un problema del medico, oggi è anche un problema del malato. Quindi, evidenza scientifica "e" evidenze personali.

\section{Giustapposizioni e relazioni}

L'evidenza scientifica non sarà mai sufficientemente evidente se non è anche convincente. Essa non sarà mai così convincente se oltre a solide basi oggettive e a buone conoscenze, non ha relazioni. La clinica nasce come un sapere osservazionale irrelato, cioè senza relazioni, concepito sulla base delle evidenze scientifiche disponibili. $\grave{E}$ del tutto insensato pensare di avere relazioni con un'evidenza scientifica, con un organo, con una malattia, con un corpo malato. Per avere relazioni si deve per forza considerare l'evidenza scientifica della malattia come propria di una persona. Solo con le persone si hanno relazioni.

Tuttavia, sostenere che la clinica non ha relazioni non è del tutto corretto, anche perché comunque un rapporto con il malato, con i suoi familiari, inevitabilmente esiste. È più corretto dire che la clinica non ha relazioni tali da influenzare la sua razionalità e le sue evidenze scientifiche. Essa ha però giustapposizioni, cioè un genere di rapporti che permette all'evidenza scientifica di non essere inquinata da qualcosa che non sia rigorosamente organico, fisico, sostanziale [11]. 
"Giustapporre" significa "porre a fianco", "mettere accanto", "accostare"; "giustapposizione" indica un "rapporto di semplice contiguità"; "giustapposto" è qualcosa che sta accanto, accostato. Si tratta di rapporti senza interdipendenze, che quindi permettono all'evidenza scientifica di essere indipendente, autonoma, autogiustificativa rispetto a tutto quello che non è considerato scientifico. La giustapposizione si basa sui ruoli e non sulle relazioni. Il ruolo del medico è giustapposto al ruolo del malato e viceversa. L'evidenza scientifica è giustapposta all'opinione personale. Si tratta della vecchia distinzione tra episteme e doxa, tra scienza e opinione, sulla quale anche la scienza medica si è basata, e per la quale la prima vale molto, la seconda non vale nulla.

La giustapposizione è la distanza/vicinanza tra due posizioni corrispondenti. L'ideale della giustapposizione clinica, quindi, non è né dialogico, né condividente, né partecipante, ma è razionale, impersonale, prescrittivo. L'evidenza scientifica ha tutte queste caratteristiche. Essa è una dimostrazione di oggettività, di fattualità, di obiettività. Tali caratteristiche nella relazione con l'altro sono destinate a "complessificarsi".

\section{Evidenza clinica $e$ relazione}

Evidenza clinica e relazione implicano un ripensamento delle giustapposizioni:

- giustapposizioni e relazioni sono due modi diversi di conoscere clinicamente la malattia e il malato;

- le relazioni sono soprattutto un problema di conoscenza, non di amabilità;

- giustapposizioni e relazioni sono due modi diversi di intendere l'evidenza scientifica. In un caso essa è una conoscenza autosufficiente, nell'altro essa è comunque una conoscenza interdipendente;

- giustapposizioni e relazioni danno luogo a organizzazioni della medicina, quindi a organizzazioni delle discipline scientifiche e degli operatori molto diverse. La prima preferirà schemi tayloristici, dunque divisionali; la seconda schemi integrati, dunque dipartimentali.

Oggi una clinica priva di relazioni è considerata socialmente inaccettabile. L'evidenza scientifica della clinica, oltreché vera, oggettiva, fattuale, deve essere anche plausibile e ragionevole:

- se l'evidenza scientifica deriva dalla razionalità scientifica $e$ dalla relazione, allora essa non è più solo una conoscenza biologica, ma è una conoscenza biologica estesa. Non è più solo una proprietà della malattia da conoscere, ma a sua volta diventa una relazione tra il malato da conoscere e il soggetto che conosce;

- se l'evidenza scientifica è una conoscenza biologica estesa, allora è una conoscenza complessa del corpo, della malattia, del malato, della situazione, del contesto, è una conoscenza delle credenze, delle conoscenze e delle convinzioni del medico che crede nelle evidenze riscontrate;

- se l'evidenza scientifica è una conoscenza complessa, essa pone la metodologia nella relazione e pone la relazione nella metodologia. Si tratta, cioè, di mettere nella relazione le giustificazioni epistemiche che riguardano l'evidenza clinica;
- se l'evidenza clinica è il risultato dell'incontro tra metodologia e relazione, allora essa è il risultato dell'incontro tra giustificazioni epistemiche e credenze e opinioni personali. Se essa è l'incontro tra oggettività, fattualità, verità scientifica, credenze del medico e opinioni del malato, decade la separazione tra osservatore e osservato, tra soggettivo e oggettivo, tra conoscenze e credenze. Se è una riunificazione del sapere in relazione a un malato, essa si propone come un ripensamento del discorso sulla cura;

- se l'evidenza scientifica è ripensamento della cura, diventerà una funzione a molti argomenti (biologici, psichici, personali, culturali, scientifici, situazionali). Se è intesa come funzione di cura a molti argomenti, allora essa accrescerà le sue convenienze sociali, scientifiche, economiche. Se è più conveniente, allora vi saranno meno contenzioso legale, meno medicina difensiva, più appropriatezza, meno incongruità terapeutiche, più capacità e abilità professionali, più corresponsabilizzazione e condivisione da parte dei cittadini, più tolleranza nei confronti della fallibilità medica. .. più beni morali, scientifici e materiali.

\section{Clinica relazionale $e$ ragionevole}

La clinica e la relazione, cioè il mondo biologico e la relazione, definiscono la clinica come "una conoscenza a molte conoscenze" [11] in cui il biologico è immerso in una complessità fatta da un contesto, un essere e la persona. La clinica relazionale è una forma evoluta di clinica in cui la relazione non è quella di cui in genere si parla nei convegni, cioè banalmente una questione di amabilità deontologica, ma è il condizionale attraverso il quale avviene una complessificazione della conoscenza della malattia. La relazione, proprio per questo, ha importanti effetti di rinnovamento e di ripensamento della clinica ortodossa da almeno tre punti di vista:

- gnoseologico, perché cambia e arricchisce la conoscenza scientifica;

- epistemologico, perché cambia il modo di conoscere della clinica attraverso la complementarità tra verità scientifica e opinione;

- metodologico, perché cambia il valore e l'uso delle procedure. Non una libertà del clinico dalla procedura, ma una libertà del clinico nella procedura.

In sostanza, mentre l'EBM è una procedura che fa dipendere la scelta del clinico da un'evidenza dogmatica, la clinica relazionale è funzione della scelta del clinico e del malato, quindi una vera e propria medicina della scelta ragionevole. Ragionevole è il rendersi conto, da parte del clinico, dell'esistenza di una complessità. Ragionevole è rendersi conto che non esiste solo la razionalità e che la razionalità è sollecitata dalla complessità ad acquisire soprattutto un nuovo senso pratico. Oggi la clinica ha il problema della persuasione: i suoi argomenti devono convincere una molteplicità di persone, i malati e i loro familiari, gli amministratori della sanità assillati dalla parità di bilancio, la società civile che ormai esprime una domanda di qualità delle cure che non ha precedenti. La clinica, e quindi i clinici, dovranno creare le condizioni per il consenso, l'assenso, la condivisione delle loro scelte. Oggi le questioni più importanti che ruotano intorno al rapporto tra complessità e clinica riguardano sempre più il problema dei trattamenti, delle decisioni 
cliniche, dell'impiego delle tecnologie, della personalizzazione delle cure. Oltre a questo l'economia pone alla clinica inediti problemi di ragionevolezza clinica (rapporto costi/ benefici, appropriatezza, economicità e antieconomicità, qualità, contenzioso legale, medicina difensivistica ecc.).

Quindi la ragionevolezza clinica, oltreché rifarsi, com'è ovvio, alle evidenze scientifiche, è quasi costretta a essere adeguata non solo ai soggetti, ma anche alle situazioni. L'evidenza scientifica pragmaticamente non può sottrarsi alla sua complessificazione. Essa è ragionevolmente adeguata se non trascura i limiti e le possibilità che vi sono in una situazione. Il malato, nella clinica relazionale e ragionevole, diviene la misura della razionalità clinica, non il contrario. Solo se la razionalità clinica è adeguata alle relazioni di complessità essa è ragionevole [12]. Essere ragionevoli vuol dire non solo avere conoscenze, capacità, ma anche sensibilità, quindi non solo avere competenze, ma anche bravure. Il ragionevole è una forma di intelligenza, di pensiero, un atteggiamento pragmatico, un modo di conoscere, un atteggiamento mentale. Per un clinico, essere ragionevole significa non essere dogmatici, quindi stare dentro la complessità con le proprie evidenze scientifiche, certo, ma pensate con un pensiero flessibile.

\section{Conflitto di interessi}

Gli autori non hanno nessun conflitto di interessi.

\section{Bibliografia}

[1] Nardi R, Fabbri T, Belmonte G, Leandri P, Mazzetti M, Pasquale $A$, et al. Medicina interna, paziente complesso, evidence based medicine e le non evidenze. Ital J Med 2009;3:191-200.

[2] Cavicchi I. L'uomo inguaribile. In: Il significato della medicina. Roma: Editori Riuniti; 1998.

[3] Cavicchi I. Il rimedio e la cura. In: Cultura terapeutica tra scienza e libertà. Roma: Editori Riuniti; 1999.

[4] Cavicchi I. La medicina della scelta. Torino: Bollati Boringhieri; 2000.

[5] Cavicchi I. Verso una pediatria dell'evidenza scientifica e della relazione. In: Principi N, Rubino A, Vierucci A (a cura di). Pediatria generale specialistica. Milano: Casa Editrice Ambrosiana; 2012.

[6] Cavicchi I. Filosofia della pratica medica. Torino: Bollati Boringhieri; 2002.

[7] Cavicchi I. La clinica e la relazione. Torino: Bollati Boringhieri; 2004.

[8] Cavicchi I. Ripensare la medicina. In: Restauri, reinterpretazioni, aggiornamenti. Torino: Bollati Boringhieri; 2005.

[9] Cavicchi I. Filosofia della medicina. AA VV Enciclopedia filosofica, vol. 7. Bompiani: Milano; 2006.

[10] Cavicchi I. Medicina e sanità: snodi cruciali. Bari: Dedalo; 2010.

[11] Cavicchi I. La relazione di cura. In: Battaglia L, Carpanelli I, Tuveri G (a cura di). Etica della cura in oncologia. Roma: Carocci; 2010.

[12] Cavicchi I. Una filosofia per la medicina. In: Razionalità clinica tra attualità e ragionevolezza. Bari: Dedalo; 2011. 